\title{
CIENCIA\&SALUD
}

\section{Enfoque práctico del paciente con Ictericia.}

\section{Practical approach of the icteric patient.}

Maria Angelica Montero Rojas ${ }^{1}$ Andrea Peña Montenegro² Luis Felipe Loaiza Sáenz ${ }^{3}$ Kevin Josué Oviedo Pérez ${ }^{4}$ Breidy Baltodano Torres ${ }^{5}$

1,2,3,4 y 5 Médico general. Trabajador independiente, San José Costa Rica.

Contacto: mari.montero.rojas@hotmail.com

\section{RESUMEN}

La ictericia, patología sumamente común en cualquier escenario clínico, se da porque la bilirrubina al ser un metabolito de la descomposición del grupo Heme que debe ser metabolizado en el hígado y ser parte de la absorción de ciertos nutrientes en el tracto intestinal, presenta muchos puntos donde su metabolismo o función se puede ver afectada. Existe una gran gama de posibles etiologías para su aparición, en las cuales la edad, estilo de vida y comorbilidades del paciente deben ser tomados en cuenta. Una historia clínica minuciosa de síntomas actuales, antecedentes familiares, medicamentos utilizados, entre otros, es esencial en conjunto con un examen físico detallado, enfocándose en signos directos e indirectos de alguna patología causante.

Los exámenes de gabinete como las enzimas hepáticas, pruebas de coagulación y anticuerpos virales apoyan las sospechas clínicas presentadas. De igual manera, el avance de la tecnología y mayor disponibilidad de ultrasonidos, tomografía y resonancia magnética facilitan el diagnóstico en estos pacientes. Lo anterior presenta una mayor facilidad y rapidez al realizar el tratamiento correspondiente a estos pacientes.

\section{Cómo citar:}

Montero Rojas, M. A., Peña Montenegro, A., Loaiza Sáenz, L. F. ., \& Oviedo Pérez, K. J. Enfoque Práctico del Paciente con Ictericia. Revista Ciencia Y Salud Integrando Conocimientos, 5(5), Pág. 19-26. https:// doi.org/10.34192/ cienciaysalud.v5i5.295

Recibido: 08/Abr/2021 Aceptado: 04/Oct/2021 Publicado: $18 /$ oct $/ 2021$
Palabras Clave: Ictericia, Hiperbilirrubinemia, Fisiología, Secreción Biliar, Hiperbilirrubinemia Conjugada,Hiperbilirrubinemia No conjugada.

\section{ABSTRACT}

Jaundice, extremely common pathology in any clinical scenario, bilirubin being a metabolite of the decomposition of the Heme group that must be metabolized in the liver and be part of the absorption of nutrients in the intestinal tract, it presents many pathways where its metabolism or function may be affected. There is a wide range of possible etiologies when it occurs, where the patients age, lifestyle and comorbidities of the patient must be taken into account. A thorough medical history of current symptoms, family history, medications used, among others, is essential in conjunction with a detailed physical examination, not only looking for direct but indirect signs of any probable pathology. The different tests that support clinical suspicions such as liver enzymes, coagulation tests and viral antibodies, as well as the improvement with technology and greater availability of ultrasound, tomography 


\section{CIENCIA\&SALUD}

and magnetic resonance imaging may facilitate diagnosis in these patients, thus giving greater ease and speed within treatment.

Keywords: Jaundice, Hyperbilirubinemia, Physiology, Bile secretion, Conjugated hyperbilirubinemia, Unconjugated hyperbilirubinemia

\section{INTRODUCCIÓN}

La ictericia conocida también como hiperbilirrubinemia es una decoloración amarillenta de la piel resultante de un exceso de bilirrubina. El rango normal de bilirrubina total es de $0.0 \mathrm{mg} / \mathrm{dL}$ a $1.0 \mathrm{mg} / \mathrm{dL}$ y la ictericia se vuelve aparente cuando sus niveles alcanzan al menos el doble de este rango (1-3). Esta patología puede resultar de distintos orígenes. Se podrían valorar tres sitios específicos en los cuales al sufrir una noxa pueden generar ictericia.

Con respecto de la ictericia pre hepática secundaria a un aumento de hemolisis, su etiología puede ser tanto congénita (talasemias, esferocitosis, etc) o adquirida como en la hemolisis autoinmune, transfusiones sanguíneas o trauma. Se vuelve importarte recalcar que la clínica variará significativamente secundaria a la patología causante de la destrucción eritrocitaria.

En el caso de la ictericia hepática, se reconocen como causas los desórdenes adquiridos o congénitos que provocan una extracción alterada de la bilirrubina plasmática, por lo que se conjuga y excreta erróneamente. Además de lo anterior, puede existir una afectación directa los hepatocitos, ya sea de etiología viral o medicamentosa. Algunos ejemplos de causas congénitas de ictericia hepática se mencionan los síndromes de Crigler Najjar, Gilbert's, Rotor's, Dubin Johnson, entre otros.

Con respecto a la ictericia extra hepática o ictericia obstructiva, esta última es la más común en la práctica quirúrgica, causada por interferencia en la salida del flujo del sistema biliar, por la obstrucción del lumen del árbol biliar. Usualmente es secundaria a coledocolitiasis, pero también se presentan malignidades de la propia vía o de órganos a su alrededor, causando así compresión $(4,5)$.

\section{Metabolismo de la Bilirrubina}

La bilis es una solución acuosa producida y secretada por el hígado. Aproximadamente $80 \%$ se deriva del metabolismo de la hemoglobina y se encuentra compuesta por fosfolípidos, colesterol, sales biliares, electrolitos, agua y bilirrubina conjugada(BC) $(6,7)$. Se requiere de múltiples protagonistas en la producción y secreción de la bilirrubina como lo son los colangiocitos, hepatocitos y vías biliares permeables para su transporte (6). El proceso de producción se lleva a cabo en las células reticuloendoteliales, células fagociticas (Kuffer), donde se realiza el quebrantamiento de moléculas de heme presentes en la hemoglobina, mioglobina, citocromos, catalasas y triptófanos; presentando como resultado el producto metabólico llamado bilirrubina (7). El grupo heme es convertido a biliverdina mediante la reacción de catalización de la oxigenasa heme mononuclear y posteriormente sufre una reducción adicional mediante la reductasa de biliverdina para conseguir el producto inicial, la bilirrubina no conjugada (BNC). Adicionalmente se conjuga con el glucuronato para crear bilirrubina conjugada en el hígado. La excreción de la bilirrubina requiere que esta se encuentre en estado hidrofílico mediante el quebrantamiento de enlaces de hidrógeno a través de la conjugación de ácido glucurónico de las cadenas de ácido propiónico de la bilirrubina (7).

Una característica importante de la BNC es que esta se une a la albúmina plasmática y a proteínas de bajo peso para su transporte, esto se cree previene la salida de la bilirrubina desde el espacio intravascular. Dado que es una molécula polar e lipofílica la convierte en la responsable por toxicidades como el Kernicterus (7).

\section{Manejo del Paciente Ictérico}

\section{Historia Clínica}

Los pacientes con ictericia, en su mayoría, consultan por medio del servicio de emergencias, se debe 


\section{CIENCIA\&SALUD}

hacer una historia clínica con el objetivo de identificar posible etiología de la ictericia, manifestaciones de hiperbilirrubinemia, órganos comprometidos y complicaciones, además de los siguientes pasos a tomar en la evaluación del mismo $(1,2)$.

Estos son algunos síntomas que los paciente pueden presentar, sin embargo no se limitan a: coloración amarillenta de la piel, prurito, alteración del estado mental, fatiga, anorexia, vómitos, pérdida no intencional de peso, aumento de peso dado el edema, distensión abdominal, diarrea, dolor abdominal, sensación febril, entre otras. Sin embargo, los pacientes que presentan un aumento de BNC pueden presentar los síntomas mencionados o asintomáticos durante un examen de rutina $(7,8)$.

Como se ha mencionado anteriormente, la historia de la patología actual debe incluir detalles minuciosos como inicio del cuadro, duración, si el mismo ha sido fluctuante o en aumento y progresión, dado que los síntomas pueden llegar a ser inespecíficos, además de historia familiar relevante con posibles causas hereditarias (hemocromatosis, enfermedad de Wilson, anemias hemolíticas) $(4,7)$.

La presentación de ciertos síntomas hace que algunas patologías sean de mayor probabilidad: dolor abdominal de predominio en cuadrante superior derecho sugiere enfermedad colestásica, también si es acompañado al dolor abdominal y/o el paciente indica fiebre, puede estar señalando un proceso infeccioso. La pérdida de peso involuntaria puede indicar cuadro de malignidad o proceso crónico $(4,7)$.

El estilo de vida también debe ser interrogado en personas jóvenes presentándose con una historia de pocos días de presentación se asocia mayoritariamente con hepatitis virales, conductas de riesgo como el uso de drogas intravenosas, experiencias sexuales casuales o viajes recientes, hacen que la hepatitis $B$ sea la causa más probable; hepatitis A, C y E se presentan rara vez en estos pacientes (5). Se debe indagar en medicamentos prescritos, naturales o drogas, ya que la lista de estos productos que afectan el metabolismo de la bilirrubina es variada, incluyendo los medicamentos que llegan a hacer hepatotóxicos (9).

La Hepatitis alcohólica es un diagnóstico clínico que se define como: inicio de ictericia dentro de los 60 días de consumo de alcohol excesivo (más de $50 \mathrm{~g} /$ dia) por un mínimo de 6 meses, con bilirrubinas mayores a $3 \mathrm{mg} / \mathrm{dL}$, AST elevada 50-400, radio AST/ALT mayor a 1.5 y sin hallazgos de otra causa de hepatitis $(4,10)$. La historia de consumo de alcohol con tanta frecuencia y cantidad se vuelve importante dado que el daño hepático por dicha sustancia es de la etiología más común en la población (5). El consumo excesivo de alcohol puede provocar hígado graso, esteatosis, hepatitis alcohólica y eventualmente cirrosis. La hepatitis alcohólica se caracteriza por inicio súbito de ictericia, hepatomegalia y signos sugestivos de respuesta inflamatoria sistémica.

\section{Hallazgos al examen físico}

El examen físico debe incluir peso y estatura para calcular el índice de masa corporal. La manifestación clínica de la ictericia representada por coloración amarillenta de la esclera (ictericia escleral) se vuelve evidente cuando los niveles de bilirrubina exceden $3 \mathrm{mg} / \mathrm{dl}$ (11). El abdomen debe ser cuidadosamente examinado para la posible identificación de masas y hepato-esplenomegalia. Pacientes con dolor localizado en hipocondrio derecho a la inspiración profunda (signo de Murphy) pueden indicar colecistitis aguda, la ley de Courvoisier indica que si un paciente presenta ictericia, la vesícula biliar es palpable y se encuentra distendida, la ictericia es poco probable que sea causada por un lito. Se deben buscar signos y estigma de enfermedad hepática crónica, entre ellos: eritema palmar, contractura Dupytren, asterixis, ginecomastia, cabeza medusa, ascitis y edema (4).

Otros hallazgos físicos que pueden guiar el diagnóstico incluyen: decoloración grisácea de la piel en hemocromatosis, anillos de Kayser Fleischer en la enfermedad de Wilson. A partir del hallazgo de ganglio supraclavicular izquierdo (nódulo de Virchow) se debe sospechar de malignidad $(9,12)$.

Es importante considerar diagnósticos diferenciales, los cuales incluyen anemia hemolítica, reabsorción de hematomas y carotenemia, de los cuales su abordaje y discusión no serán abordados en este artículo, sin embargo esta última es importante distinguirla dado que se debe en un aumento de la coloración 


\section{CIENCIA\&SALUD}

amarillenta de la piel pero sin aumento de bilirrubina. Asimismo, tampoco presenta coloración amarillenta de escaleras limitándose a áreas como manos y pies (8).

Laboratorio y Gabinete

En la evaluación del paciente con ictericia, los laboratorios determinan ciertos patrones que dejan en evidencia dónde puede estar la etiología subyacente (13), además de la bilirrubina, se deben realizar los laboratorios comúnmente llamados pruebas de función hepática, a pesar de que no se encuentran relacionadas directamente con su funcionamiento (9). Las pruebas incluyen la fosfatasa alcalina (FA), alanina y aspartato aminotransferasa, (ALT y AST, respectivamente) y gamma glutamil transferasa (GGT) $(3,13)$. En general, Ios laboratorios que reflejan lesión hepática son ALT, AST, fosfatasa alcalina y bilirrubina. La función hepatocelular se puede valorar mediante marcadores como: albumina, bilirrubina y tiempo de protrombina (1).

La valoración inicial del paciente con ictericia se basa en identificar si la hiperbilirrubinemia es resultado de bilirrubina conjugada o no conjugada (3), esto permite facilitar la identificación de la causa de la ictericia, es decir, prehepática, hepática o posthepática (9). Adicionalmente es adecuado realizar un hemograma para la evaluación y detección de anemia y presencia de hemólisis o hiperesplenismo con trombocitopenia (7). Asimismo, es de frecuente uso la valoración de distintos marcadores y estudios complementarios como:

- Fosfatasa alcalina: producida principalmente en el hígado, pero también se encuentra en hueso y en poca cantidad en intestinos, riñón y células blancas. Hay aumentos fisiológicos de la FA asociados a crecimiento óseo y en embarazo (producido por la placenta), y en patologías asociado a enfermedad colestásica, obstrucción de conductos biliar común, colangitis biliar primaria, colestasis intrahepática o colestasis por medicamentos. Cuando solo se encuentra elevada la FA, el medir la GGT puede determinar si el aumento de FA es de origen hepático o no (12).

- GGT: abundante en el hígado, presentes en otros órganos pero no el hueso, por esto se usa para diferenciar el origen de la FA. Los aumentos de la GGT se dan por obesidad, exceso de alcohol e inducción medicamentosa. A pesar de la poca especificidad, es uno de los mejores predictores de mortalidad hepática (12).

- AST y ALT: enzimas presentes en los hepatocitos liberadas en caso de daño celular. ALT es la enzima considerada más hepato específica en caso de inflamación, en comparación con AST, al ser producida por los hepatocitos y encontrarse predominantemente en el hígado. En comparación, la AST se encuentra presente en concentraciones importantes, no solo en hígado, sino en músculo esquelético, cardíaco y liso; a pesar de esto, la AST es más sensible como indicador de lesión hepática relacionada con alcohol (12). Concentraciones elevadas de aminotransferasas de más de $1000 \mathrm{U} / \mathrm{L}$ se presentan en lesiones hepáticas agudas con daño celular típicas de isquemia, secundario a toxinas hepáticas o comúnmente hepatitis virales agudas. La elevación de estas enzimas y su patrón dan una sospecha clínica sobre los posibles diagnósticos, si sus valores de AST son menores a 8 veces, el valor normal y ALT es menor de 5 veces. El diganóstico diferencial posible es hepatitis alcohólica; si ambas se encuentran menos de 4 veces del valor normal, es posible enfermedad grasa hepática no alcohólica, en valores extremos como ya mencionamos antes, más de 25 veces se sospecha de hepatitis aguda o relacionada con toxinas y más de 50 veces los diagnósticos más probables con isquemia o shock hepático (1). Es importante recalcar que en casos de enfermedad hepática crónica estos marcadores presentan menor relevancia dado que pueden estar elevados o pueden encontrarse en valores normales, secundario a un mínimo de células sanas en el parénquima $(7,11)$.

- Bilirrubinuria: es definida como la presencia de bilirrubina en orina identificada por urianalisis, su presencia es lógicamente anormal y podría ser el primer indicador de enfermedad seria hepatobiliar antes de que la ictericia clínica aparezca. La BNC presenta solubilidad en grasa pero no en agua por lo 


\section{CIENCIA\&SALIUD}

que no puede ser excretada mediante el riñón, por lo tanto, la presencia de bilirrubina en orina indica un predominio de bilirrubina conjugada (14).

- El hemograma es beneficioso para la determinación de hemólisis, el cual se caracteriza por daño en células rojas y aumento de reticulocitos. Como se mencionó anteriormente, es importante recordar que un aumento mayor a $3 \mathrm{mg} / \mathrm{dl}$ de bilirrubina total (BT) ocasiona que se torne clínicamente aparente. La hiperbilirrubinemia es de predominio no conjugado cuando la bilirrubina conjugada es menor al $15 \%$ de la BT. En contraste con la hiperbilirrubinemia conjugada, la BC es mayor a $20 \%$ de la BT $(7,8)$.

La hiperbilirrubinemia puede también ser causada por infecciones, enfermedades autoinmunes y desórdenes genéticos, por esto son útiles laboratorios como serología de hepatitis, estudios del hierro o anticuerpos en búsqueda de estas patologías $(4,15)$.

\section{Estudio de Imagen}

Después de la evaluación del paciente con lo anteriormente mencionado, realizar un ultrasonido transabdominal es de vital importancia, entre los estudios que se pueden realizar, este es poco costoso y no invasivo. Al realizarlo, se deben buscar datos de dilatación intra o extrahepática, vesícula biliar distendida o contraída y presencia de litos tanto en la vesícula como en el árbol biliar. La presencia de enfermedad neoplásica extrahepática causa dilatación biliar detectable, también se deben describir las características del hígado, parénquima y ecogenicidad, si hay presencia de lesiones sólidas o quísticas. En la obstrucción por litos biliares por la naturaleza intermitente del cuadro, puede presentar un ultrasonido normal, pero una clínica altamente sugestiva sugiere realizar otros estudios $(4,5)$.

El ultrasnido con elastografía transitoria (Fibroscan) es usado para medir el grado de rigidez del parénquima hepático, es ventajoso sobre la biopsia al ser aún un procedimiento no invasivo (5). Otro tipo de ultrasonido que se puede realizar es un ultrasonido endoscopio, utilizado en su mayoría para el estadiaje de masas periampulares (4).

EI TAC con doble medio de contraste puede mostrar la dilatación de la vía biliar y el nivel de la obstrucción, además de posibles cambios de enfermedad hepático crónica como ascitis, varices y eplenomegalia. Entre otros estudios de imagen se encuentran la resonancia magnética colangiopancreatografia y colanggiopancreatografía retrograda, la primera ha empezado a reemplazar a la segunda, al detallar el árbol biliar sin ser invasivo (4).

Estos estudios se valorarán para la búsqueda de dilatación en el árbol biliar, tumores, litos o datos falla hepática.

\section{TRATAMIENTO}

La hiperbilirrubinemia es una manifestación de una gran gama de enfermedades más que una enfermedad aislada propiamente. El tratamiento es variable según su etiología, todos los medicamentos hepatotóxicos deben ser suspendidos hasta determinar la causa de la hiperbilirrubinemia conjugada, dado que estos pueden producir progresión del daño hepático (7). Si la posible etiología se trata de una hepatitis alcohólica, la piedra angular del tratamiento se basa en ingesta nutricional adecuada y abstinencia. Aproximadamente el $10-20 \%$ de pacientes evoluciona a cirrosis en contraste con un 10\% que presentan regresión del daño hepático (10).

En la hepatitis alcohólica existen modelos para determinar la severidad de la hepatitis en búsqueda de determinar que los pacientes se favorecerán de una intervención farmacológica. El factor discriminante de Maddrey (MDF) incluye bilirrubina sérica total y protrombina con el objetivo de segregar pacientes con mortalidad mayor a 50\% en los próximos 28 días. Los pacientes con MDF mayor a 32 son considerados a beneficiarse con el uso de terapia esteroidea. La escala de Lille obtiene datos del principio y final de la primera semana de la terapia esteroidea para valorar respuesta y necesidad subsecuente de tratamiento. 
Con respecto a estos pacientes con hepatitis alcohólica severa con o sin encefalopatía, son candidatos a un curso corto de prednisolona (40 mg/día por 28 días). Se vuelve importante mencionar que la prednisolona es preferida a la prednisona, dado que no requiere metabolismo hepático para su actividad, sin embargo siempre deben tomarse en cuenta contraindicaciones para uso de esteroides (ej: sangrado gastrointestinal, pancreatitis severa, diabetes no controlada, infección activa o insuficiencia renal). La abstinencia y la adecuada ingesta nutricional es el pilar del tratamiento de la hepatitis alcohólica, aproximadamente un 10\% de estos individuos presenta regresión de la lesión hepática con la abstinencia (10).

En casos de colelitiasis donde los pacientes no son candidatos a cirugía o declinan la cirugía se puede considerar el uso de sales biliares orales (ácido ursodeoxicólico $10 \mathrm{mg} / \mathrm{kg} / \mathrm{día}$ ), los cuales reducen la secreción biliar de colesterol, provocando la disolución de litos de colesterol. Otra alternativa constituye la litotripsia extracorpórea por ondas de choque, sin embargo cuenta con contraindicación en casos de colecistitis, pancreatitis biliar, coledocolitiasis, embarazo y coagulopatías. Es importante recalcar que los pacientes tratados como los anteriormente mencionados presentan recurrencia de litos en un $50 \%$ versus 70\% respectivamente (7).

\section{CONCLUSIONES}

Se vuelve de vital importancia frente a un paciente que presenta clínicamente ictericia realizar un trabajo en equipo interprofesional para utilizar los recursos, completar estudios y lograr identificación adecuada de la etiología causante y así ofrecerle el mejor tratamiento para evitar posibles complicaciones, morbilidad y mortalidad relacionada con el cuadro. Igualmente, es fundamental la importancia de la comunicación abierta entre el médico y el paciente para generar conciencia de las enfermedades prevenibles como la hepatitis y alcoholismo, asimismo como educación a pacientes portadores de antecedentes heredofamiliares de cáncer hepatobiliar para reconocer signos y síntomas tempranos de ictericia asociada.

\section{REFERENCIAS BIBIOGRÁFICAS}

1. Friedman L. Approach to the patient with abnormal liver biochemical and function tests [Internet]. UpToDate. 2020 [citado el 3 de Febrero 2021]. Disponible en: https://www.uptodate.com/contents/ approach-to-the-patient-with-abnormal-liver-biochemical-and-function-tests

2. Roy-Chowdhury N, Roy-Chowdhury J. Diagnostic approach to the adult with jaundice or asymptomatic hyperbilirubinemia [Internet]. UpToDate. 2020 [citado el 3 de Febrero 2021]. Disponible: hhttps://www.uptodate.com/contents/diagnostic-approach-to-the-adult-with-jaundice-or-asymptomatichyperbilirubinemia

3. Roy-Chowdhury N, Roy-Chowdhury J. Classification and causes of jaundice or asymptomatic hyperbilirubinemia [Internet]. UpToDate. 2020 [citado el 3 de Febrero 2021]. Disponible en: https://www. uptodate.com/contents/classification-and-causes-of-jaundice-or-asymptomatic-hyperbilirubinemia

4. Novo C, Welsh F. Jaundice [Internet]. ScienceDirect. 2017 [citado el 15 de Febrero 2021]. Disponible en: https://www.sciencedirect.com/science/article/abs/pii/s026393191730220X

5. Patanwala I, Richardson P, Garvey C, Gilmore I. Jaundice in the acute setting [Internet]. ScienceDirect. 2016 [citado el 15 de Febrero 2021]. Disponible: https://doi.org/10.1016/j.mpmed.2016.11.008

6. Hundt M, Basit H, John S. Physiology, Bile Secretion [Internet]. Ncbi.nlm.nih.gov. 2021 [citado 2 Abril 2021]. Disponible en: https://www.ncbi.nlm.nih.gov/books/NBK470209/

7. Tripathi N, Jialal I. Conjugated Hyperbilirubinemia [Internet]. Ncbi.nlm.nih.gov. 2021 [citado 2 Abirl 2021]. Disponible en: https://www.ncbi.nlm.nih.gov/books/NBK562172/

8. Singh A, Jialal I. Unconjugated Hyperbilirubinemia [Internet]. Ncbi.nlm.nih.gov. 2021 [citado 2 Abril 2021]. Disponible en: https://www.ncbi.nlm.nih.gov/books/NBK549796/ 


\section{CIENCIA\&SALUD}

9. Vagholkar K. Obstructive Jaundice: Understanding the pathophysiology [Internet]. ResearchGate. 2020 [citado el 3 Febrero del 2021];6(4):26-31. Disponible en: https://www.researchgate.net/ publication/343617705_Obstructive_Jaundice_Understanding_the_pathophysiology

10. Shah N, Royer A, John S. Alcoholic Hepatitis [Internet]. Ncbi.nlm.nih.gov. 2021 [citado 2 Abril 2021]. Disponible en: https://www.ncbi.nlm.nih.gov/books/NBK470217/

11. Joseph A, Samant H. Jaundice [Internet]. Ncbi.nlm.nih.gov. 2021 [cited 2 April 2021]. Available from: https://www.ncbi.nlm.nih.gov/books/NBK544252/

12. Ravindran R. Jaundice [Internet]. Surgery Oxford. 2020 [citado el 22 de Febrero 2021];6(4):26-31. Disponible en: https://doi.org/10.1016/j.mpsur.2020.06.008

13. Gondal B, Aronsohn A. A Systematic Approach to Patients with Jaundice [Internet]. Pubmed. 2016 [citado el 15 de Febrero 2021]. Disponible en: https://pubmed.ncbi.nlm.nih.gov/27904243/

14. Hoilat G, John S. Bilirubinuria [Internet]. Ncbi.nlm.nih.gov. 2021 [citado 2 Abril 2021]. Disponible en: https://www.ncbi.nlm.nih.gov/books/NBK557439/

15. Mizrahi M, Lalazar G, Horwich Y, Adar T, Safadi R. Benign recurrent intrahepatic cholestasis type-II--a rare cause of direct hyperbilirubinemia exacerbations with hepatic fibrosis [Internet]. PubMed. 2021 [citado 2 Abril 2021]. Disponible en: https://pubmed.ncbi.nlm.nih.gov/18770956/ 